\title{
AVALIAÇÃO DA MATURAÇÃO DO PROCESSO DE RACIOCÍNIO DIAGNÓSTICO DE ESTUDANTES DE MEDICINA
}

\author{
AN APPRAISAL OF THE MATURATION OF MEDICAL STUDENTS’ DIAGNOSTIC ABILITY
}

Dejano T. Sobral

Professor Adjunto da Faculdade de Medicina da Universidade de Brasília

Correspondência: D.T. Sobral - Universidade de Brasília - C.P. 04569 - Medicina. 70919-970 Brasília - DF. Tel.: (0xx) 61-3072269 Fax: (0xx) 61-5772268 - E-mail: dtsobral@unb.br

SOBRAL DT. Avaliação da maturação do processo de raciocínio diagnóstico de estudantes de Medicina.

Medicina, Ribeirão Preto, 34: 89-99, jan./mar. 2001.

RESUMO: O propósito do estudo foi averiguar a utilidade do Inventário de Raciocínio Diagnóstico (IRD) na avaliação da maturação do processo de diagnóstico clínico, em conexão com percepções sobre o aprendizado na formação médica. O inventário foi aplicado entre 88 estudantes de Medicina, de ambos os sexos, em dois semestres consecutivos na fase de iniciação clínica. Foram, também, obtidos dados de percepções do aprendizado (valorização do aprendizado, autoconfiança do aprendiz e reflexão na aprendizagem) e de rendimento acadêmico. Os resultados revelaram diferenças significantes, porém pequenas, entre as duas medidas dos escores do IRD e correlações positivas e significantes desses escores com pontuações das três medidas de percepção do aprendizado. Observou-se uma relação significativa entre o tamanho do efeito e a percepção de autodeterminação do processo diagnóstico. Os dados indicam que o IRD reflete, além de conhecimento e habilidades específicas, a percepção de eficácia pessoal no processo de diagnóstico, que pode ser influenciada por diferentes fatores do contexto do aprendizado e de atitudes do aprendiz. Em conclusão, os achados apontam a sensibilidade discriminativa do instrumento e sugerem sua utilidade no acompanhamento da maturação do processo de diagnóstico clínico.

UNITERMOS: Diagnóstico Clínico. Estudantes de Medicina. Educação Médica. Atitude. Inventário de Raciocínio Diagnóstico.

\section{INTRODUÇÃO}

O diagnóstico clínico é um processo complexo, que constitui um aspecto distintivo da expertise em diferentes campos de atuação profissional, o que realça o interesse do estudo da diferenciação da capacidade de diagnóstico, durante a formação médica. Estudantes e profissionais parecem utilizar processos de raciocínio diagnóstico similares, em sua essência, segundo estudos realizados por diferentes investigadores que usaram, por vezes, técnicas complexas e de aplicação demorada.
Bordage, Grant \& Marsden, 1990 ${ }^{(1)}$, desenvolveram o Inventário de Raciocínio Diagnóstico (IRD) para aferir a capacidade de diagnóstico clínico. O instrumento pode ser respondido em 15-20 minutos, facilitando sua utilização no contexto dos estudos em Medicina. Os investigadores descreveram duas categorias de processo: grau de flexibilidade no raciocínio e grau de estrutura do conhecimento na memória, com base em numerosos elementos de pesquisa ${ }^{(1 / 5)}$. O estudo original revelou variância e discriminação nos escores do instrumento. Foram observadas, por exemplo, diferenças significantes entre médias de grupos 
representativos de fases distintas da educação médica. Trabalhos subseqüentes confirmaram a validade de conteúdo, mostraram a estabilidade temporal e indicaram a utilidade potencial do instrumento na apreciação do raciocínio diagnóstico, em diferentes circunstâncias ${ }^{(6 / 9)}$.

Estudos realizados com estudantes brasileiros revelaram que características dos aprendizes e fatores do contexto educativo influenciavam a resposta ao inventário $^{(6,10)}$. Foram observadas associações significativas entre o tipo de experiência prévia do aprendiz e as medidas de processo identificadas por Bordage $\mathrm{e}$ colegas. Observaram-se, ainda ,correlações positivas entre os escores do inventário e medidas de rendimento cognitivo. Em contraponto, aspectos de atitudes na aprendizagem - tais como o nível de autoconfiança como aprendiz - também apresentaram associações relevantes com o escore do inventário.

Os achados citados foram reforçados em novo estudo de validação, cujos resultados sugerem que o IRD reflete, além de conhecimento e habilidades específicas, a noção de capacidade pessoal no processo de diagnóstico, que pode ser influenciada por diferentes fatores do contexto do aprendizado e de atitudes do aprendiz (Sobral, não publicado). Essa relação pode ser interpretada em termos do princípio da autoeficácia exposto por Bandura, $1997^{(11)}$. Segundo esse investigador, a percepção da auto-eficácia refere-se às crenças pessoais na capacidade de organizar e efetuar os atos requeridos para conseguir uma realização e tem caráter preditor em relação ao funcionamento psicossocial do indivíduo.

Qual é a utilidade do IRD na apreciação da maturação da capacidade de diagnóstico? Essa questão foi examinada no presente estudo, que faz parte de uma linha de investigação sobre o desenvolvimento do processo de diagnóstico, realizado na situação real da aprendizagem clínica de um curso de Medicina. Nessa perspectiva, o instrumento de Bordage e colegas foi aplicado a uma mesma amostra de estudantes de graduação, em dois semestres distintos do curso, e os resultados foram analisados com referência aos fatores identificados nos trabalhos referidos. Três objetivos são realçados a seguir.

1) Descrever os escores do IRD e suas diferenças na amostra pareada de estudantes.

2) Relacionar diferenças nos escores com a percepção de autodeterminação no processo de diagnóstico e outras características dos estudantes.
3) Averiguar a força preditora relativa do escore total do IRD em relação à capacidade de resolução de problemas clínicos.

\section{METODOLOGIA}

Sujeitos - A população alvo do estudo compreendia estudantes do curso de Medicina da Universidade de Brasília. O critério de inclusão foi a conclusão do sexto semestre do curso, na amostragem consecutiva de seis turmas de ingresso semestral. Do total de 243 sujeitos incluídos (59,7\% masculinos), 88 (52,3\% masculinos) constituíram o grupo de estudo, por terem cursado disciplina eletiva específica no quinto semestre. Os $2 / 3$ restantes formaram o grupo-controle. O número total da amostra representa $60 \%$ do alunado do curso no período do estudo.

Contexto - A coleta de dados foi efetuada em dois momentos do curso: no quinto semestre, quando se dá a iniciação clínica em Semiologia, e no sexto semestre, quando os estudantes lidam diretamente com pacientes, realizam exames clínicos completos e são orientados a desenvolver o diagnóstico clínico nas enfermarias de clínica médica e clínica cirúrgica. $\mathrm{O}$ intervalo entre as duas medidas foi da ordem de cinco meses, mas corresponde a um tempo de experiência clínica de 12-14 semanas.

Medidas e Procedimentos - Três descritores da situação do aprendiz foram aplicados à amostra total no terceiro semestre do curso: (a) inventário de valorização do aprendizado, de Nehari \& Bender, $1978^{(12)}$; (b) escala analógica visual de autoconfiança ${ }^{(13)}$; e (c) índice de rendimento acadêmico, calculado pela média ponderada das menções nas disciplinas correspondentes. O inventário de valorização do aprendizado retrata as percepções do aprendiz sobre os desfechos da aprendizagem (abrangendo tanto componentes de conteúdo quanto de desenvolvimento pessoal), em termos de valor e significado para o respondente. As respostas podem refletir as influências e limitações do contexto educativo na visão do aprendiz e estão associadas aos níveis de satisfação e prazer sentidos na aprendizagem. O índice de confiabilidade (alfa de Cronbach, consistência interna) foi 0,90 na amostra total.

Um quarto descritor, a escala de reflexão na aprendizagem $^{(14)}$, foi aplicado no quinto semestre para os participantes do grupo de estudo. Essa escala revelou-se útil na apreciação da influência do papel da reflexão na aprendizagem, especialmente em termos 
de integração e monitoração da aprendizagem, requeridas no aprendizado profundo. O índice de consistência interna, no caso, foi 0,86 .

O Inventário de Raciocínio Diagnóstico (IRD), na versão em Português, foi aplicado no quinto semestre ( $1^{\text {a }}$ medida) e no sexto ( $2^{\text {a }}$ medida). O IRD tem 41 itens, dentre os quais 21 medem a flexibilidade de diagnóstico. Cada item contém um enunciado seguido de uma escala de seis pontos, do tipo diferencial, semântica. Esse instrumento procura captar diversas facetas do processo diagnóstico clínico, entre as quais: aspectos salientes, reinterpretação de achados, modo de inquirir, mudança de percepção, julgamento reversível, relações abstratas e acesso à estrutura do conhecimento na memória. O índice de consistência interna, na amostra total, foi 0,82 . A estabilidade temporal em duas semanas (teste-reteste), obtida em subamostra, foi satisfatória $(0,77)$.

O IRD foi aplicado com referência ao modo geral de raciocínio diagnóstico, tendo em conta a natureza da experiência clínica dos participantes e o propósito de exercício educativo. Na aplicação do inventário, incluiu-se, adicionalmente, uma questão expressa em escala global, de quatro pontos, para aferir a percepção de autodeterminação no raciocínio diagnóstico. A extensão de autodeterminação, na escala, se estendia de restrita (necessitando amadurecimento, incentivo, treinamento e feedback para desenvolvimento de raciocínio clínico eficaz), até máxima (capaz de raciocínio clínico eficaz mesmo em condições adversas de tempo e contexto de desempenho). Variantes da escala têm relação estreita com índices de auto-eficácia no desempenho acadêmico (Sobral, não publicado) .

O último instrumento usado foi um teste de resolução de problemas clínicos, que consta de 15 casos clínicos variados, em 60 questões, e foi aplicado após a segunda medida do IRD, no sexto semestre. O teste tem elevada validade de conteúdo e moderada validade preditora, em relação ao desempenho cumulativo do estudante no curso.

Análise - Os textos de Norman \& Streiner, $1994^{(15)}$, e Hulley \& Cummings, $1988^{(16)}$, orientaram os planos e procedimentos de análise. Três procedimentos estatísticos principais foram utilizados: (a) coeficientes de correlação para medir a associação entre pares de variáveis; (b) testes t, ou análises de variância, para aferir diferenças entre médias de grupos; (c) análise de regressão múltipla para verificar a associação entre fatores explanatórios e a variável de desfecho, quando outros fatores são controlados. A análise foi realizada no programa SPSS 9.0.

\section{RESULTADOS}

As médias dos escores do IRD e de fatores antecedentes (valorização do aprendizado, autoconfiança como aprendiz e rendimento acadêmico) não foram significativamente diferentes na comparação entre os grupos de estudo e controle. Não houve, também, diferenças significantes entre participantes agrupados por gênero ou coorte. A média do escore total do IRD no sexto semestre, no conjunto dos estudantes, foi 170,31 (desvio padrão 17,91), correspondendo a $69 \%$ da pontuação máxima do instrumento.

A Tabela I mostra os escores do IRD na primeira e na segunda medidas dos participantes do grupo de estudo. Observou-se correlação positiva e significante entre as duas medidas. A diferença média entre as medidas foi significante, para cada escore. $\mathrm{O}$ tamanho do efeito ('effect size') foi da ordem de 0,4. Cerca de $67 \%$ dos participantes apresentaram elevação do escore total, após o treinamento clínico do sexto semestre.

\begin{tabular}{|c|c|c|c|c|c|c|}
\hline \multirow[t]{2}{*}{ Escores do IRD } & \multirow{2}{*}{$\frac{1^{\mathrm{a}} \text { Medida }}{\text { Média }(\mathrm{dp})}$} & \multirow{2}{*}{$\begin{array}{c}2^{\mathrm{a}} \text { Medida } \\
\text { Média }(\mathrm{dp})\end{array}$} & \multicolumn{2}{|c|}{ Correlação } & \multicolumn{2}{|c|}{ Teste t } \\
\hline & & & $r$ & $p$ & Diferença & $p$ \\
\hline Flexibilidade no raciocínio & $\begin{array}{l}85,48 \\
(8,94)\end{array}$ & $\begin{array}{l}88,73 \\
(8,56)\end{array}$ & 0,51 & 0,000 & 3,25 & 0,001 \\
\hline Estrutura de conhecimento & $\begin{array}{l}81,88 \\
(8,70)\end{array}$ & $\begin{array}{l}85,33 \\
(8,89)\end{array}$ & 0,52 & 0,000 & 3,45 & $<0,001$ \\
\hline Total & $\begin{array}{c}167,35 \\
(15,81)\end{array}$ & $\begin{array}{l}174,10 \\
(16,04)\end{array}$ & 0,57 & 0,000 & 6,75 & $<0,001$ \\
\hline
\end{tabular}

*A versão do instrumento, que foi utilizada no estudo, consta de anexo. 
As relações entre o IRD e fatores antecedentes (rendimento cognitivo, autoconfiança como aprendiz, reflexão na aprendizagem e valorização do aprendizado) foram analisadas por coeficientes de correlação. A Tabela II mostra as correlações entre o escore total do IRD, no sexto semestre, e os antecedentes apurados. As três medidas subjetivas (valorização do aprendizado, autoconfiança como aprendiz e reflexão na aprendizagem) mostraram correlação positiva e significante com o escore total do IRD.

Verificou-se que os escores do IRD eram significativamente distintos, na dependência do nível de percepção da autodeterminação no processo de raciocínio diagnóstico. A Tabela III revela as diferenças entre as duas medidas dos escores do IRD para participantes agrupados segundo nível baixo (restrito ou parcial) ou nível alto (amplo ou máximo) de autodeterminação nesse processo. As diferenças entre escores foram pouco expressivas no subgrupo de baixo nível de autodeterminação e bem significativas no subgrupo

\begin{tabular}{|lrrc|}
\hline $\begin{array}{l}\text { Tabela II - Coeficientes de correlação do escore total do IRD no sexto semestre, } \\
\text { com fatores antecedentes de percepção do aprendizado e rendimento acadêmico } \\
\text { (N=88) }\end{array}$ & Médias (dp) & $\begin{array}{c}\text { Correlação } \\
\text { com IRD }\end{array}$ & $\mathrm{p}$ \\
\hline Fatores apurados no $5^{\circ}$ semestre & $82,9(15,8)$ & 0,31 & $<0,001$ \\
\hline Autoconfiança como aprendiz & $117,7(12,9)$ & 0,24 & $<0,001$ \\
Valorização do aprendizado & $52,22(6,66)$ & 0,41 & $<0,001$ \\
Reflexão na aprendizagem & $3,73(0,43)$ & 0,16 & 0,155 \\
Rendimento no $5^{\circ}$ semestre & $85,48(8,94)$ & 0,53 & $<0,001$ \\
Flexibilidade no raciocínio & $81,88(8,70)$ & 0,48 & $<0,001$ \\
\hline Estrutura do conhecimento na memória & & & \\
\hline
\end{tabular}

de alto nível. O tamanho do efeito, neste segundo subgrupo, foi 0,6 .

Obteve-se uma análise de regressão múltipla em que a segunda medida do escore total do IRD é a variável de desfecho e as variáveis preditoras são as diversas medidas ou fatores antecedentes: pontuações de autoconfiança como aprendiz, valorização do aprendizado, reflexão na aprendizagem, rendimento acadêmico, escores da primeira medida de flexibilidade de raciocínio e estrutura de conhecimento na memória no quinto semestre, bem como nível de autodeterminação no processo de diagnóstico. Três fatores explanatórios, nível de autodeterminação, flexibilidade no raciocínio e estrutura do conhecimento na memória foram identificados. O coeficiente $\mathrm{R}$ de correlação múltipla foi 0,67 . Isso significa que essas três variáveis respondem por $45 \%$ da variância do escore do IRD no sexto semestre. A Tabela IV mostra os diferentes coeficientes da análise de regressão.

Por fim, obteve-se uma análise de regressão múltipla para aferir a eventual contribuição do IRD na explicação da variância do rendimento no teste de resolução de problemas clínicos, como variável de desfecho, controlando-se o efeito de todas as outras medidas antecedentes. O modelo de regressão múltipla (stepwise) identificou três fatores preditores, em ordem decrescente de contribuição: rendimento no terceiro semestre, escore de estrutura do conhecimento na memória (do IRD) e rendimento no quinto semestre. $\mathrm{O}$ coeficiente $\mathrm{R}$ de

\begin{tabular}{|c|c|c|c|c|c|c|}
\hline \multirow{3}{*}{$\begin{array}{c}\text { Escores do IRD } \\
\text { Flexibilidade no raciocínio }\end{array}$} & \multicolumn{2}{|c|}{$\begin{array}{l}\text { Nível de autodeterminação } \\
\text { Baixo (restrito/parcial) }\end{array}$} & \multirow{3}{*}{$\begin{array}{c}\text { Teste } \mathrm{t} \\
\mathrm{p} \\
0,041\end{array}$} & \multicolumn{2}{|c|}{$\begin{array}{l}\text { Nível de autodeterminação } \\
\text { Alto (amplo/máximo) }\end{array}$} & \multirow{3}{*}{$\begin{array}{c}\text { Teste } t \\
p \\
0,002\end{array}$} \\
\hline & $1^{\mathrm{a}}$ Medida & $2^{\mathrm{a}}$ Medida & & $1^{\mathrm{a}}$ Medida & $2^{\mathrm{a}}$ Medida & \\
\hline & $(7,63)$ & 84,49 & & $88,62 \quad(9,07)$ & $92,92 \quad(7,73)$ & \\
\hline Estrutura de conhecimento & $(8,08)$ & 80,68 & 0,224 & 84,13 & $90,23 \quad(8,16)$ & $<0,001$ \\
\hline Total & $160,46(13,66)$ & $165,20 \quad(13,81)$ & 0,061 & $172,74(16,07)$ & $183,23(14,28)$ & $<0,001$ \\
\hline
\end{tabular}


correlação múltipla foi 0,57 . A Tabela $\mathrm{V}$ mostra que o escore de estrutura do conhecimento na memória foi um fator preditor significante, embora fraco, da capacidade de resolução de problemas clínicos ao término do semestre.

\section{DISCUSSÃO}

Os achados deste e de outros estudos devem ser considerados na perspectiva de que os estudantes diferem em seu entendimento do processo de diagnóstico e na própria orientação de aprendizagem desse processo, o que influencia os desfechos de qualquer medida da capacidade de diagnóstico ${ }^{(17)}$. Os níveis de correlação entre respostas da primeira e da segunda medida sugerem que uma parcela dos estudantes têm uma percepção instável da consistência das condutas do processo de diagnóstico, incorporadas no instrumento de Bordage e colegas.

O tamanho do efeito (effect size) da intervenção (experiência clínica) nos escores do IRD foi pequeno $(0,4)$. Três possibilidades, não excludentes, podem ser aventadas: o grau limitado de experiência clínica no intervalo entre as medidas, a baixa transferência do conhecimento clínico, acumulado no período para a situação do teste, e a própria percepção do nível de autodeterminação, no processo de diagnóstico, entretida pelos participantes. No subgrupo com alto nível de autodeterminação, o tamanho do efeito foi moderado $(0,6)$.

A análise de regressão múltipla sugere que a contribuição do nível de autodeterminação, no processo de diagnóstico, como fator explicativo do escore total do IRD, parece incorporar os efeitos da percepção de valorização ou significado do aprendizado, do grau de autoconfiança do aprendiz e de seu nível de reflexão na aprendizagem.

Uma possibilidade postulada para interpretar essa contribuição do nível de autodeterminação - ten- do em conta a própria natureza dessa medida - baseia-se no conceito da auto-eficácia ${ }^{(11)}$. No caso, a expectativa de auto-eficácia se refere às convicções pessoais do estudante quanto a sua competência em organizar e efetuar as condutas requeridas para a consecução do processo de diagnóstico. São fontes de informação para a percepção da auto-eficácia as experiências diretas ou vicariantes, a persuasão verbal e o estado afetivo ou fisiológico do aprendiz. Esses elementos são inerentes ao contexto de uso do processo de diagnóstico nas condições de aprendizagem, na iniciação clínica da formação médica.

Nesse sentido, um componente subjacente das respostas ao IRD pode ser a percepção mantida por cada participante quanto ao nível e à intensidade de sua eficácia pessoal na maturação e no uso do processo de diagnóstico clínico. A associação entre escores do IRD e de reflexão na aprendizagem reforça essa inferência, na medida em que a auto-reflexão medeia a construção da percepção de eficácia pessoal, a partir das informações de diferentes fontes obtidas pelo aprendiz ${ }^{(18)}$.

A correlação positiva e significante, observada entre o IRD e a pontuação do teste de resolução de 
problemas, confirmou indicações anteriores e representa um indício da validade de critério do instrumento, considerando a importância do processo de diagnóstico no teste clínico. Em acréscimo, os dados da análise de regressão múltipla sugerem que o processo aferido pelo IRD é um fator explanatório independente, embora fraco, de rendimento cognitivo na iniciação clínica. Esse fator está possivelmente associado ao componente de percepção de eficácia pessoal nas diferentes facetas do processo de diagnóstico, captadas pelo instrumento.

No conjunto, as relações observadas indicam a sensibilidade discriminativa do Inventário de Raciocínio Diagnóstico e permitem inferências sobre a validade de construto do IRD. A perspectiva do processo de diagnóstico, aferida na medida do IRD, parece refletir, além de informações e habilidades adquiridas na formação, a percepção de eficácia pessoal no processo de diagnóstico e o perfil de conduta individual (em termos de interação interpessoal, motivação e processo decisório) no encontro clínico. Essa idéia implica a ocorrência de múltiplas fontes de variação nas respostas ao inventário, tais como o níveis de autodeterminação e de reflexão na aprendizagem, exemplificadas no estudo. Meyer \& Cleary, $1998^{(16)}$, usando outro tipo de instrumento, identificaram 11 fontes de variação na noção e no engajamento de estudantes em relação ao diagnóstico clínico.

Os achados têm implicação para a interpretação dos escores do IRD, especialmente no seguimen- to longitudinal do processo de desenvolvimento da capacidade de diagnóstico. A identificação de diferentes perfis de resposta pode servir para acompanhamento e orientação dos aprendizes, no ensino clínico, quanto aos componentes do processo de diagnóstico, seja flexibilidade no raciocínio (reinterpretação de achados, modo de inquirir, mudança de percepção e julgamento reversível) ou conhecimento funcional (aspectos salientes, relações abstratas e acesso à estrutura do conhecimento na memória). Uma alteração significativa, positiva ou negativa, nos escores, pode refletir mudança na percepção de eficácia pessoal, associada a fatores do aprendiz ou do contexto de treinamento.

Em suma, este estudo examinou as relações e distribuição de escores do Inventário de Raciocínio Diagnóstico, numa amostra representativa da população alvo de estudantes, inseridos no contexto real de aprendizagem clínica. Os resultados confirmam a utilidade do inventário para captar, de forma rápida, o modo de diagnóstico utilizado pelo respondente, em função de seu contexto atual e da experiência prévia. Os achados também sugerem a conveniência do uso do instrumento para acompanhamento da maturação do processo de diagnóstico clínico.

\section{AGRADECIMENTOS}

$\mathrm{O}$ autor agradece a participação ativa e o interesse real dos aprendizes envolvidos.

SOBRAL DT. An appraisal of the maturation of medical students' diagnostic ability. Medicina, Ribeirão Preto, 34: 89-99, jan./march 2001.

ABSTRACT: The purpose of this study was to assess the utility of the Diagnostic Thinking Inventory (DTI) for appraisal of diagnostic ability development in relation to medical students' learning perceptions. The inventory was administered to 88 medical students of both sexes, before and after regular clinical studies, five-month apart. Different measures of students' learning perceptions (self-confidence as a learner, meaningfulness of learning experience and reflection in learning) and academic achievement were also obtained. The results showed significant albeit small differences between the two DTI measures as well as positive significant correlation of measures of learning perceptions with DTI scores. There was a significant relationship between the effect size of the intervention and the perception of self-determination in the diagnostic process. The data reinforce the idea that the DTI scores reflect, in addition to knowledge and skills, the learners' perceptions of personal efficacy in the diagnostic process. Such perceptions can be influenced by many factors of the learning context as well as the learners' attitudes to learning. In conclusion, the findings indicate the discrimination sensibility of the instrument and suggest its usefulness in monitoring the maturation of the clinical diagnostic process.

UNITERMS: Diagnosis, Clinical. Students, Medical. Education, Medical. Attitude. Diagnostic Thinking Inventory. 


\section{REFERÊNCIAS BIBLIOGRÁFICAS}

1 - BORDAGE G; GRANT J \& MARSDEN P. Quantitative assessment of diagnostic ability. Med Educ 24: 413-425, 1990.

2 - BORDAGE G \& ZACKS R. The structure of medical knowledge in the memory of medical students and general practitioners: categories and prototypes. Med Educ 18: 406-411, 1984.

3 - GALE J \& MARSDEN P. Medical diagnosis: from student to clinician. Oxford University Press, Oxford, 1983.

4 - GRANT J \& MARSDEN P. The structure of memorised knowledge in students and clinicians: an explanation for diagnostic expertise. Med Educ 21: 92-98, 1987.

5 - LEMIEUX M \& BORDAGE G. Structuralisme et pédagogie médicale: étude comparative des stratégies cognitives d'apprentis-cliniciens. Rech Sém 6: 143-179, 1986.

6 - SOBRAL DT. Diagnostic ability of medical students in relation to their characteristics and preclinical background. Med Educ 29: 278-282, 1995.

7 - JONES UF. The reliability and validity of the Bordage, Grant \& Marsden inventory for use with physiotherapists. Med Teacher 19: 133-140, 1997.

8 - PETERSON C. Factors associated with success or failure in radiological interpretation: diagnostic-thinking approaches. Med Educ 33: 251-259, 1999.

9 - ROUND AP. Teaching clinical reasoning - a preliminary controlled study. Med Educ 33: 480-483, 1999.
10 - SOBRAL DT. Appraisal of medical students' diagnostic ability in relation to their learning achievement and self-confidence as a learner. Med Teacher 22: 59-63, 2000.

11 - BANDURA A. The nature and structure of self-efficacy. In: BANDURA A. Self-efficacy. The exercise of control. W.H. Freeman, New York, p. 36-78, 1997.

12 - NEHARI M \& BENDER $\mathrm{H}$. Meaningfulness of a learning experience: a measure for educational outcomes in higher education. Higher Educ 7: 1-11, 1978.

13 - SOBRAL DT. Motivação para aprender e resultados da aprendizagem baseada em problemas. Psicol: Teoria Pesq 9: 555-562, 1993.

14 - SOBRAL DT. Desenvolvimento e validação de escala de reflexão na aprendizagem. Psicol: Teoria Pesq 14: 173177, 1998.

15 - NORMAN GR \& STREINER DI. Biostatistics: the bare essentials. Mosby, St. Louis, 1994.

16 - HULLEY SB \& CUMMINGS SR. Designing clinical research. Williams and Wilkins, Baltimore, 1988.

17 - MEYER JHF \& CLEARY EG. An exploratory student learning model of clinical diagnosis. Med Educ 32: 574-581, 1998.

18 - SOBRAL DT. An appraisal of medical students' reflectionin-learning. Med Educ 34: 182-187, 2000.

Recebido para publicação em 07/08/2000.

Aprovado para publicação em 17/01/2001. 


\section{A N EXO}

\section{LIDANDO COM PROBLEMAS DIAGNÓSTICOS}

Orientação. Este inventário* contém 41 itens referentes ao raciocínio diagnóstico em Medicina. Cada item consta de um enunciado, duas afirmações coligadas e uma escala de resposta entre as afirmações. A escala representa um continuum entre as duas afirmações opostas.

Faça, por favor, um X naquele espaço :__: dentre os marcos da escala que melhor descreva sua posição nesse continuum.
Inexistem respostas corretas ou erradas. Responda da forma mais espontânea que puder, indicando sua provável conduta na situação descrita - isto é, aquela que acontece(ria) mais freqüentemente (ainda que não tenha experiência específica da situação). Escolha sempre entre as alternativas dadas, mesmo que possam existir outras opções.

O inventário leva 15 a 20 minutos para completar. Em retorno, você receberá um comentário escrito dentro de duas semanas.

\author{
01 - Quando o paciente apresenta seus sintomas, \\ penso nos sintomas e nas \\ palavras exatas usadas pelo \\ paciente.
}

penso nos sintomas em termos mais abstratos do que as expressões usadas (exemplo: "4 dias de duração" torna-se "agudo").

tento dar a cada um igual importância ou peso.

primeiro, coleto as informações clínicas e, então, penso a respeito.

usualmente, acho fácil explorar vários diagnósticos possíveis.

muitas vezes, uma idéia sobre a natureza do problema se fixa na minha mente

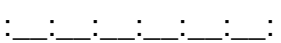

diagnóstico bem no início, na

04 - Quando estou entrevistando um paciente,

05 - Do princípio ao fim de uma entrevista (anamnese),

se eu sigo a linha de idéias do paciente, a tendência é que perca minha própria linha.

\section{6 - Quando se trata de resolver-me acerca de um diagnóstico,}

não me importo em adiar minhas

decisões de diagnóstico sobre

um caso.

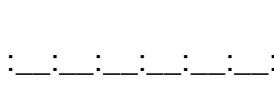

sinto-me obrigado a concluir por um diagnóstico ou outro, mesmo que não esteja muito seguro.

mesmo que siga a linha de idéias do paciente eu ainda posso manter claras minhas próprias idéias.

07 - Logo que o paciente tenha apresentado claramente os seus sintomas e sinais,

penso sobre eles na minha mente nas próprias palavras do paciente.

traduzo-os mentalmente por termos médicos (exemplo: "formigamento" vira parestesia).

\section{8 - Em relação à história clínica de rotina,}

freqüentemente sinto que não dei abrangência suficiente à anamnese de rotina.

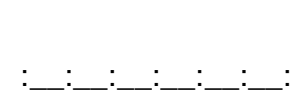

usualmente, completo a anamnese de rotina de modo satisfatório para mim.

\footnotetext{
"Versão do instrumento de Bordage, Grant \& Marsden (1990). D.T. Sobral, Universidade de Brasília.
} 


\section{9 - Enquanto o paciente conta sua história e o caso se revela,}

muitas vezes, tenho dificuldade

em relembrar o que foi dito. usualmente, posso manter em minha mente o que foi dito.

\section{0 - Durante o desenrolar de uma entrevista, acho que}

alguns itens-chave de

informação parecem saltar aos

meus olhos.

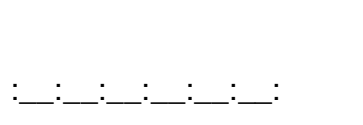

muitas vezes, é difícil saber em quais itens de informação se agarrar.

\section{1 - Quando não consigo fazer sentido dos sintomas do paciente,}

passo adiante e obtenho mais

informações para despertar novas idéias. peço ao paciente para definir tais sintomas mais claramente.

\section{2- Ao considerar possibilidades de diagnóstico em casos clínicos,}

muitas vezes, apresento

diagnósticos improváveis. habitualmente, estou na pista certa.

\section{3 - Quando estou obtendo informações sobre um paciente,}

os vários itens de informação parecem se agrupar, por si, juntos na minha mente.

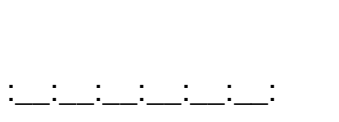

muitas vezes tenho dificuldades em ver como os itens de informação se relacionam entre si.

14 - Quando o diagnóstico torna-se conhecido e descubro quem, inicialmente, ele me escapou,

muitas vezes é porque eu conhecia a doença, mas faltoume pensar nela. muitas vezes é porque eu não conhecia o suficiente sobre a doença.

\section{5 - Durante a entrevista clínica,}

não posso convencer-me a descartar alguma informação como irrelevante.

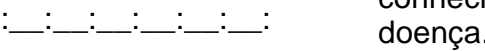

\section{6 - Quando não consigo fazer sentido dos sintomas e sinais do paciente,}

passo a obter novas informações

e uma nova perspectiva. examino-os sob perspectiva diferente antes de prosseguir.

\section{7 - Quando considero um número de possíveis diagnósticos (hipóteses),}

as hipóteses tendem a se

relacionar entre si. as hipóteses tendem a ser dispersas.

\section{8 - Quando um possível diagnóstico surge na minha mente, durante a entrevista,}

usualmente, vejo-me

antecipando, mentalmente, possíveis sintomas e sinais que combinam com tal hipótese. bem freqüentemente, essa hipótese não me ajuda a decidir o que perguntar ao paciente, a seguir.

19 - Quando conheço bem pouco sobre um tipo particular de doença,

ainda posso, usualmente, propor um diagnóstico. tenho muita dificuldade em propor um diagnóstico.

\section{0 - Ao considerar os sinais e sintomas do paciente,}

penso em cada um em termos absolutos, conforme foram expressos pelo paciente. penso neles em termos de possíveis opostos (e.g., progressivo vs. súbito; espástico vs. flácido; unilateral vs. bilateral). 
21 - Quando sei muito sobre um tipo particular de doença e tenho que fazer um diagnóstico, acho relativamente fácil chegar a uma conclusão de diagnóstico.

muitas vezes, pareço dispersivo e tenho dificuldade em chegar a uma conclusão.

22 - Com a evolução da história e já tendo algumas idéias sobre diagnóstico(s) possível (eis), novas informações, freqüentemente, me fazem ter Novas informações não me fazem, mais idéias. freqüentemente, ter mais idéias.

23 - Quando estou tirando uma história, acho que

posso obter novas idéias por simples repasse dos dados existentes na minha mente.

preciso ter novos dados para que venha a ter uma nova idéia sobre o caso.

24 - Quando o paciente usa expressões imprecisas ou ambíguas, deixo que ele prossiga para manter o fluxo da entrevista.

faço com que ele esclareça precisamente o que quer dizer, antes de prosseguir.

\section{5 - Depois de uma entrevista com um paciente,}

tenho poucas outras questões

que deveria ter perguntado, face

ao distúrbio do paciente. tenho muitas outras questões que deveria ter perguntado, face ao distúrbio do paciente.

\section{6 - Quando um item de informação emerge e me faz pensar num possível diagnóstico,}

isso me leva, muitas vezes, a voltar aos dados prévios para ver se as coisas combinam. raramente isso me faz revisar dados que obtivera anteriormente.

27 - Em relação a um diagnóstico que eu tenha finalmente feito, usualmente, eu tenho poucas dúvidas. $::::::: \quad: \quad$ inseguro.

\section{8 - Ao tomar uma decisão final de diagnóstico,}

Decido, considerando cada diagnóstico separadamente, por seus próprios méritos.

Decido, comparando e contrastando os vários possíveis diagnósticos.

29 - Quando sei muito a respeito de um tipo particular de doença e tenho que fazer um diagnóstico, verifico a maioria das possibilidades antes de chegar a uma decisão. idéias que não exploro mais extensamente.

\section{0 - À medida que o caso clínico se desdobra,}

não acho útil resumir o caso enquanto prossigo.

Periodicamente, avalio os dados e minhas idéias.

\section{1 - Quando chego às minhas decisões de diagnóstico,} muitas vezes, há informações restantes que eu simplesmente esqueci.

usualmente, terei levado em conta todas as informações.

\section{2 - Quando tenho uma idéia sobre o problema do paciente,}

sinto-me mais confortável ,se puder explorar essa idéia sem ser desviado.

$: \quad: \quad: \quad: \quad: \quad:$

sinto-me à vontade para desviar o rumo e retornar às minhas idéias mais tarde.

33 - Quando me vem uma idéia geral sobre o problema do paciente,

posso, usualmente, passar para um diagnóstico específico. acho difícil colocá-la em termos específicos. 
34 - Do princípio ao fim da entrevista,

consigo conferir minhas idéias, mesmo se deixo o paciente controlar a entrevista.

sou bem sucedido apenas se puder controlar o rumo da entrevista.

35 - No que tange à escolha entre as hipóteses de diagnóstico que tiver, usualmente, não consigo excluir totalmente qualquer das hipóteses que eu fiz.

sou capaz de descartar totalmente a maioria de minhas hipóteses.

36 - Uma vez que eu tenha chegado a uma decisão sobre um paciente, estou preparado para mudar de idéia.

eu realmente não gosto de mudar de idéia.

37 - Quando examino minhas hipóteses de diagnóstico, faço isso na base

do caso clínico por inteiro até então.

$:::::::$

38 - Se eu não souber como interpretar uma entrevista clínica, posso, prontamente, ver as informações de novas maneiras.

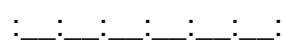

\section{9 - Quando indico exames complementares,}

faço isso como parte da investigação clínica de rotina.

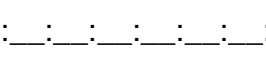

\section{0 - Ao considerar as hipóteses de diagnóstico,}

eu comparo e contrasto os possíveis diagnósticos.

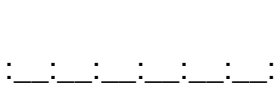

41 - Em termos do modo como eu conduzo uma entrevista, usualmente, eu rastreio a área que preciso durante a entrevista.

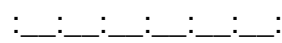

de uns poucos sintomas e sinais marcantes.

acho difícil ver as informações de novas maneiras.

faço isso na expectativa de informação específica ou evidência de apoio.

examino cada diagnóstico separadamente, por seus próprios méritos.

bem freqüentemente, não faço todas as perguntas que deveria na ocasião oportuna.

Em suma, acredito que minha eficácia pessoal no processo do raciocínio diagnóstico situa-se no seguinte nível - minha capacidade atual é:

( ) Restrita. Necessito de extensa preparação adicional (incentivo, orientação, maturação, prática e feedback) no processo de raciocínio diagnóstico.

( ) Parcial. Preciso de apoio e oportunidades de aperfeiçoamento no processo.

( ) Ampla. Tenho autonomia no processo em condições favoráveis.

( ) Máxima. Tenho plena autonomia no processo mesmo sob pressão negativa (ambiente adverso, falta de tempo ou casos difíceis).

Nome:

Matrícula: 